\title{
The Effects of Supermajority on Multi-Parent Crossover
}

\author{
Chuan-Kang Ting, Member, IEEE, and Chun-Cheng Chen
}

\begin{abstract}
Multi-parent crossover allows more than two parents participating in crossover. The increase of parents in crossover intensifies exploitation or exploration or both; however, the intensification is often unbalanced and consequently causes deterioration in performance. In this paper we propose the use of supermajority to address the issue of lopsided intensification on exploitation and exploration in multi-parent crossover. The proposed crossover, called biased occurrencebased scanning crossover (bOB), controls the tendency toward exploitation or exploration by the threshold in supermajority. Two adaptive strategies are developed to adjust the threshold of bOB. Experimental results indicate that bOB can achieve significant improvement on uniform crossover and occurrencebased scanning crossover in both solution quality and convergence speed. Precisely, the improvement in mean best fitness ranges from $4-89 \%$ on our test problems. The preferable results validate that $\mathrm{bOB}$ crossover can not only enhance the performance but also provide an effective way to control the exploitation and exploration in crossover.
\end{abstract}

\section{INTRODUCTION}

Evolutionary algorithms (EAs) have shown their effectiveness in dealing with search and optimization problems. The essence of EAs is simulating natural evolution, such as selection, crossover, and mutation to find the near-optimal solutions [7]. Crossover in EAs is ordinarily performed with two parents, by analogy with sexual reproduction in nature. However, it is not necessary to limit the number of parents in EAs. Multi-parent crossover allows more than two parents participating in the crossover operation. In terms of the number of parents, multi-parent crossover can be viewed as a generalization of traditional 2-parent crossover.

Several multi-parent crossover operators have been proposed [2], [3], [10]. In general, the increase of parents brings about a more comprehensive survey for determining the offspring genes and leads to a stronger tendency towards exploitation or exploration or both [2], [12], [13]. An effective EA, nevertheless, requires a balance of these two driving forces: Strong exploitation leads to a rapid convergence but may suffer from premature convergence, while strong exploration helps to search more regions but pays the cost of efficiency. Designing effective strategies to control the power intensified by the increase of parents is therefore a key issue in improving the performance of multi-parent crossover.

This paper presents a novel approach to address the issue of lopsided intensification on exploitation or exploration in multi-parent crossover. Specifically, we introduce the concept of supermajority into the occurrence-based scanning crossover (OB-Scan) [4] - a multi-parent generalization of uniform crossover - to replace the simple majority system

\footnotetext{
The authors are with the Department of Computer Science and Information Engineering, National Chung Cheng University, Taiwan (e-mail: ckting@cs.ccu.edu.tw; ccch95m@cs.ccu.edu.tw).
}

for determining offspring genes among multiple parents. The threshold in the supermajority affects the tendency of OB-Scan towards exploitation or exploration. Two strategies are further developed to adaptively adjust the threshold in response to the allele frequency. Experimental results show the proposed approach can effectively balance exploitation and exploration and then achieves significant improvement on OB-Scan in terms of convergence speed and solution quality on our benchmark problems.

The remainder of this paper is organized as follows. Section 2 reviews related work about multi-parent crossover. In Section 3, we detail the proposed multi-parent crossover and the derivative strategies. Section 4 presents the experimental results and performance evaluation. Finally, conclusions are drawn in Section 5.

\section{Multi-Parent Crossover}

Multi-parent crossover breaks through the convention of using two parents in crossover. The debut of multi-parent crossover in evolutionary computation was as global recombination in evolutionary strategies (ES) [1], [10]. Global recombination determines each offspring gene according to the whole population rather than only two parents. Such a global form allows an offspring to inherit from more than two parents.

For genetic algorithms (GAs), several multi-parent crossovers have been proposed. Eiben et al. proposed scanning crossover [4] and diagonal crossover [5], [6], which generalize uniform crossover [11] and one-point crossover [7], respectively. Depending on the heuristics applied to it, scanning crossover has three variations: uniform scanning crossover (U-Scan), occurrence based scanning crossover (OB-Scan), and fitness based scanning crossover (FB-Scan). Experimental results show that both scanning crossover and diagonal crossover can achieve better performance than their 2-parent versions in several test functions. Mühlenbein et al. [9], [18] introduced the concept of global recombination into GAs as gene pool recombination (GPR). Rather than from two parents, GPR samples the genes for crossover from the gene pool, which consists of several pre-selected parents. The studies indicate that GPR and its variants are easier to analyze and these methods can converge faster than 2-parent recombination. Tsutsui and Jain [16] proposed multi-cut crossover (MX) and seed crossover (SX). Multicut crossover generalizes the classic two-point crossover and was shown empirically to outperform diagonal crossover.

In addition to binary-coded GAs, Tsutsui and Ghosh [14], [15] presented three multi-parent crossover operators for realcoded GAs: center of mass crossover (CMX), multi-parent feature-wise crossover (MFX), and seed crossover (SX). 


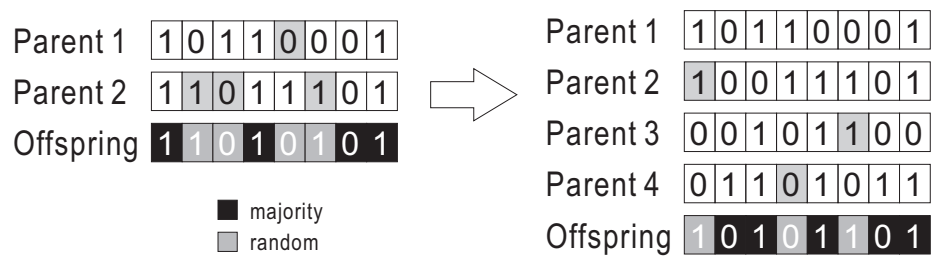

Fig. 1. Examples of 2-parent OB-Scan (left) and 4-parent OB-Scan (right)

Experimental results demonstrate that these multi-parent crossovers can lead to better performance although the performance is problem-dependent. Another multi-parent crossover, simplex crossover (SPX) [17], produces offspring on the basis of the simplex sampled from multiple parents. Their results show that SPX performs well with three or four parents for multimodal and epistatic problems. Moreover, Kita et al. [8] introduced multiple parents into unimodal normal distribution crossover (UNDX) to enhance the diversity of offspring. This multi-parent extension of UNDX exhibits its improvement in search ability on highly epistatic problems.

The theoretical work [12], [13] reveals that the increase of parents in OB-Scan intensifies the exploitation of the search. The one-sided intensification speeds up the convergence at the risk of premature convergence, which is reflected in the poor solution quality of OB-Scan using more than two parents. The present paper is to address this issue through supermajority.

\section{The PROPOSED APPROACH}

In this paper we introduce the concept of supermajority into OB-Scan to deal with the issue of unbalanced exploitation and exploration. A supermajority is a majority required to exceed a specified percentage $\theta$ higher than simple majority, e.g. $\theta=60 \%$ of total votes. Through control over the supermajority threshold $\theta$, we are able to regulate the tendency of OB-Scan towards exploitation or exploration. Before describing the approaches, we give a definition of the chromosome and the population in binary-coded GAs.

Definition 1 (Chromosome and Population):

1) a chromosome $\vec{c}$ is encoded as a bit string, i.e. $\vec{c} \stackrel{\text { def }}{=}$ $\left(c_{1}, \ldots, c_{l}\right) \in\{0,1\}^{l}$, where $c_{i}$ denotes a gene and $l$ is the chromosome length;

2) the population $C$ is a multiset of chromosomes: $C \stackrel{\text { def }}{=}$ $\left\{\vec{c}_{1}, \ldots, \vec{c}_{m} \mid \vec{c}_{i} \in\{0,1\}^{l}\right\}$, where $m$ is called the population size.

OB-Scan is a multi-parent generalization of uniform crossover. Rather than random, OB-Scan determines offspring genes depending on the occurrence of parental genes at that locus. Specifically, it takes the simple majority of parental genes as the offspring gene for each locus. Note that in this paper OB-Scan is defined to break ties by randomly ${ }^{1}$ choosing a parental gene at that locus. Figure 1

\footnotetext{
${ }^{1}$ The original OB-Scan [4] breaks ties by directly inheriting the gene from the first selected parent. However, random tie break conforms to the generalization of uniform crossover.
}

gives examples of 2-parent OB-Scan and 4-parent OB-Scan, where 2-parent OB-Scan corresponds to uniform crossover.

Definition 2 (OB-Scan): Given $n$ parents $\vec{c}_{1}, \ldots, \vec{c}_{n} \in C$, OB-Scan reproduces an offspring $\vec{c}^{\prime}=\left(c_{1}^{\prime}, \ldots, c_{l}^{\prime}\right)$ by, for $k=1, \ldots, l$,

$$
c_{k}^{\prime}= \begin{cases}0 & \frac{1}{n} \sum_{i=1}^{n} c_{i, k}<\frac{1}{2}, \\ 1 & \frac{1}{n} \sum_{i=1}^{n} c_{i, k}>\frac{1}{2}, \\ c_{r, k} & \text { otherwise, }\end{cases}
$$

where $c_{i, k}$ denotes the gene of parent $\vec{c}_{i}$ at locus $k$, and $r \in\{1, \ldots, n\}$ is generated at random.

The way that OB-Scan decides offspring genes is a "simple majority" voting in essence; that is, an allele gets elected if more than half of the total parents hold it. The theoretical analysis [12], [13] indicates that increasing the number of parents in OB-Scan leads to a higher probability for the major alleles in the population to exist in every offspring, which will cause the population to concentrate on a certain region and thus lose the diversity rapidly. This situation is so-called premature convergence, a result of unbalanced exploitation and exploration. In other words, OB-Scan using more than two parents suffers from premature convergence because it excessively intensifies exploitation but lessens exploration.

However, OB-Scan provides the capability for other voting system, namely, other criterion for determining offspring genes. In this paper we use the "supermajority" in place of the simple majority to deal with the issues in OB-Scan. The supermajority threshold $\theta$ represents the required level for the candidates to be elected. In the supermajority voting system, the winner requires a higher level $\left(\theta>\frac{1}{2}\right)$ of support than in simple majority voting system. The proposed approach is called biased occurrence-based scanning crossover $(b O B)$ in that the threshold for supermajority biases the requirement for alleles 0 and 1 to be selected. The formal definition of $\mathrm{bOB}$ is given below.

Definition 3 (bOB): Given $n$ parents $\vec{c}_{1}, \ldots, \vec{c}_{n} \in C$ and the thresholds $0 \leq \theta_{k} \leq 1$, bOB reproduces an offspring $\vec{c}^{\prime}=\left(c_{1}^{\prime}, \ldots, c_{l}^{\prime}\right)$ by, for $k=1, \ldots, l$,

$$
c_{k}^{\prime}= \begin{cases}0 & \frac{1}{n} \sum_{i=1}^{n} c_{i, k}<\theta_{k}, \\ 1 & \frac{1}{n} \sum_{i=1}^{n} c_{i, k}>\theta_{k}, \\ c_{r, k} & \text { otherwise, }\end{cases}
$$

where $c_{i, k}$ denotes the gene of parent $\vec{c}_{i}$ at locus $k$, and $r \in\{1, \ldots, n\}$ is generated at random. 
TABLE I

TEST FUNCTIONS (RAS, SCH, AND GRI)

\begin{tabular}{|c|c|c|c|c|c|}
\hline Name & Function & Range of $x_{i}$ & $N$ & Bits of $x_{i}$ & $l$ \\
\hline $\mathrm{F} 2 \mathrm{e}$ & $f_{\mathrm{F} 2 \mathrm{e}}=\sum_{i=1}^{N-1}\left[100\left(x_{i+1}-x_{i}^{2}\right)^{2}+\left(x_{i}-1\right)^{2}\right]$ & {$[-2.048,2.047]$} & 10 & 12 & 120 \\
\hline RAS & $f_{\mathrm{RAS}}=10 N+\sum_{i=1}^{N}\left[x_{i}^{2}-10 \cos \left(2 \pi x_{i}\right)\right]$ & {$[-5.12,5.11]$} & 10 & 10 & 100 \\
\hline GRI & $f_{\mathrm{GRI}}=1+\sum_{i=1}^{N} \frac{x_{i}^{2}}{4000}-\prod_{i=1}^{N} \cos \left(\frac{x_{i}}{\sqrt{i}}\right)$ & {$[-512,511]$} & 10 & 10 & 100 \\
\hline
\end{tabular}

Remark 1: Uniform crossover and OB-Scan are special cases of bOB: $n$-parent OB-Scan corresponds to $n$-parent bOB with $\theta_{k}=\frac{1}{2}$ for all $k \in\{1, \ldots, l\}$, while uniform crossover corresponds to 2-parent OB-Scan.

By definition, bOB is a general form of uniform crossover and OB-Scan. The additional parameter, $\theta_{k}$, plays an important role on the decision criterion of bOB for offspring genes. Restated, the value of $\theta_{k}$ biases bOB in favor of allele 0 or 1 at offspring locus $k$ : The case $\theta_{k}>\frac{1}{2}$ gives rise to a supermajoritarian requirement for allele 1 to be elected, which encourages bOB to choose 0 as the offspring gene. Conversely, the case $\theta_{k}<\frac{1}{2}$ brings about a supermajority for allele 0 so that bOB tends to chooses allele 1 .

In view of the above effects, we develop two strategies to control the parameter $\theta_{k}$ adaptively. The strategies are based on the allele frequency (or gene frequency), which represents the relative frequency of an allele in the population for a locus.

Definition 4 (Allele Frequency): Let $C_{k}^{(\alpha)}=\{\vec{c} \in C \mid$ $\left.c_{k}=\alpha\right\}$ be the subset in which the chromosomes possess allele $\alpha$ at locus $k$. The allele frequency is defined by

$$
p_{k}^{(\alpha)} \stackrel{\text { def }}{=} \frac{\left|C_{k}(\alpha)\right|}{|C|}
$$

where $|\cdot|$ denotes the set cardinality.

For simplicity, we refer to the allele frequency $p_{k}^{(1)}$ as $p_{k}$ and refer to $p_{k}^{(0)}$ as $\left(1-p_{k}\right)$ since binary-coded GAs hold only two types of allele frequency $p_{k}^{(1)}$ and $p_{k}^{(0)}$ with $p_{k}^{(0)}=$ $1-p_{k}^{(1)}$ for any locus $k$.

The proposed strategies for determination of threshold $\theta_{k}$ are: for all $k=1, \ldots, l$,

StRategy 1: $\theta_{k}=p_{k}$

STRATEGY 2: $\theta_{k}=1-p_{k}$

The bOB operators using the above strategies are denoted as $\operatorname{bOB}\left(n, p_{k}\right)$ and $\operatorname{bOB}\left(n, 1-p_{k}\right)$, respectively. The two strategies result in contrary effects on the determination of offspring genes: $\operatorname{bOB}\left(n, p_{k}\right)$ makes the major alleles in the population more difficult to be selected as offspring genes, while $\operatorname{bOB}\left(n, 1-p_{k}\right)$ makes them easier. That is to say, $\operatorname{bOB}\left(n, p_{k}\right)$ tends to pull the allele frequency back to the neural frequency 0.5 . This effect is expected to allow more exploration and relieve the premature convergence caused by the one-sided intensification on exploitation when using more than two parents in OB-Scan. The $\operatorname{bOB}\left(n, 1-p_{k}\right)$,
TABLE II

PARAMETERS OF GAS USED IN THE EXPERIMENTS

\begin{tabular}{|c||c|}
\hline Representation & Bit string \\
\hline GA model & Generational GA \\
\hline Population size & 100 \\
\hline Parent selection & 2-tournament \\
\hline Crossover & OB-Scan, bOB \\
\hline Number of parents & $2, \ldots, 10$ \\
\hline Crossover rate $p_{c}$ & 1.0 \\
\hline Mutation & Bit-flip \\
\hline Mutation rate $p_{m}$ & $1 / l$ \\
\hline Survivor selection & $\mu+\lambda$ \\
\hline Termination & 500 generations \\
\hline Number of runs & 100 runs per experiment \\
\hline
\end{tabular}

on the other hand, encourages the preference for the major alleles and pushes the allele frequency toward 0 or 1 , which will further increase the exploitation and thus cause an faster but more likely premature convergence than OBScan. Through $\theta_{k}$ we can control the tendency of crossover toward or against choosing major alleles as offspring genes; consequently, the exploitation and exploration in bOB can be regulated. The effects of the proposed $\operatorname{bOB}\left(n, p_{k}\right)$ and $\operatorname{bOB}\left(n, 1-p_{k}\right)$ will be thoroughly examined in the next section.

\section{Performance Evaluation}

This work conducts a series of experiments to evaluate the performance of the proposed bOB. To this end, we compare the performance of GAs using uniform crossover, $\mathrm{OB}-\mathrm{Scan}, \operatorname{bOB}\left(n, p_{k}\right)$, and $\operatorname{bOB}\left(n, 1-p_{k}\right)$, respectively. Note that 2-parent OB-Scan corresponds to uniform crossover. The effectiveness of bOB on enhancing OB-Scan will be examined in terms of solution quality and convergence speed.

The test functions include the extended De Jong's second function (F2e), the Rastrigin function (RAS), and the Griewangk function (GRI). Table I describes of these test functions and the related parameters. Note that the problem is to find the global minimum of these test functions. The setting of GAs for the experiments is summarized in Table II. Each experiment includes 100 independent runs.

\section{A. Solution Quality}

Figure 2 compares the solution quality for OB-Scan, $\operatorname{bOB}\left(n, p_{k}\right)$, and $\operatorname{bOB}\left(n, 1-p_{k}\right)$ using different number of parents. The experimental results show that, in general, 


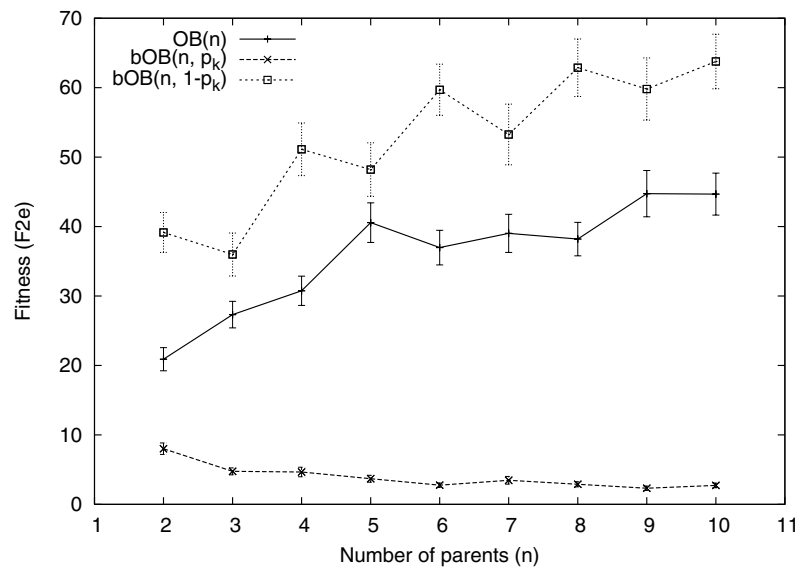

(a) $\mathrm{F} 2 \mathrm{e}$

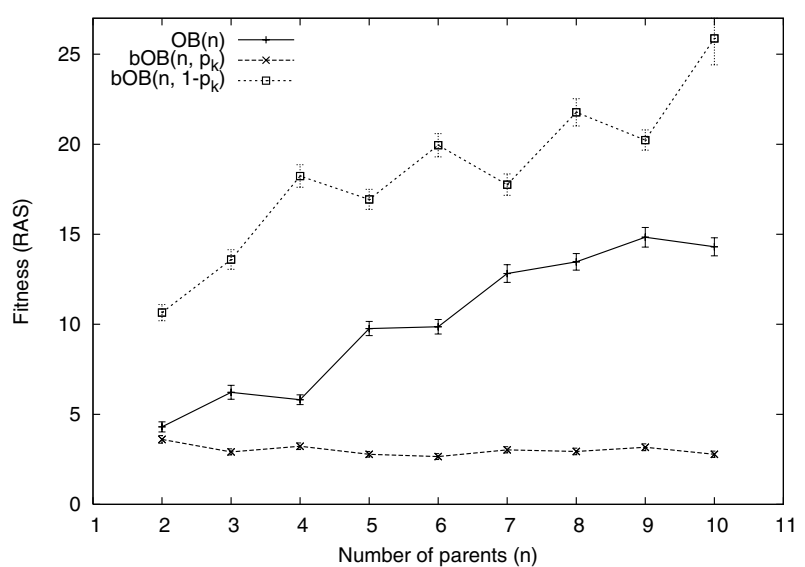

(b) RAS

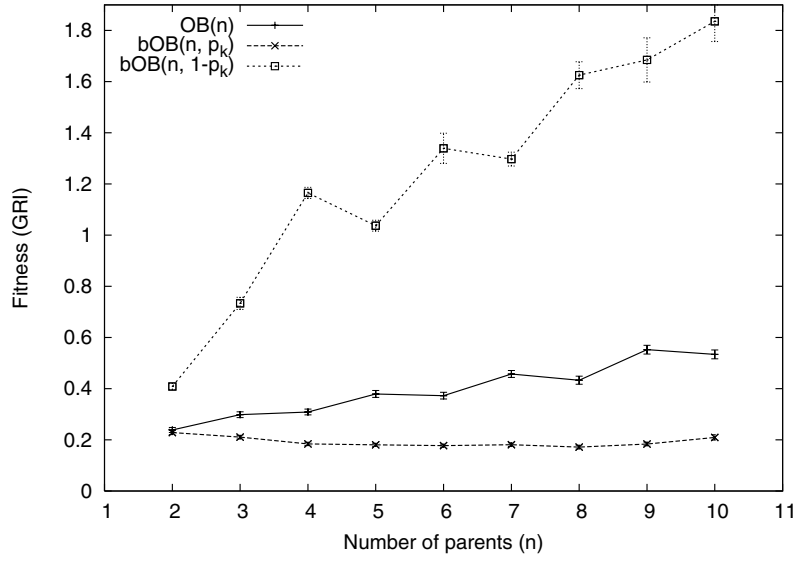

(c) GRI

Fig. 2. Mean and standard error of the best fitness for GAs using OB-Scan, $\operatorname{bOB}\left(n, p_{k}\right)$, and $\mathrm{bOB}\left(n, 1-p_{k}\right)$ with different number of parents on (a) the F2e, (b) the RAS, and (c) the GRI functions

$\operatorname{bOB}\left(n, p_{k}\right)$ outperforms OB-Scan while OB-Scan outperforms $\operatorname{bOB}\left(n, 1-p_{k}\right)$ in terms of solution quality. The preferable results of $\operatorname{bOB}\left(n, p_{k}\right)$ demonstrate its effectiveness on overcoming the issue of excessively intensified exploitation in OB-Scan. In addition, the performance of $\operatorname{bOB}\left(n, p_{k}\right)$ becomes better as the number of parents increases. On the other hand, OB-Scan and $\mathrm{bOB}\left(n, 1-p_{k}\right)$ using more than two parents perform worse than their 2-parent versions. The increase of parents worsens the resultant fitness for these two crossovers. This poor outcome reflects the problem of unbalance of exploitation and exploration in OB-Scan and especially in $\operatorname{bOB}\left(n, 1-p_{k}\right)$.

Table III accesses the improvement of $\operatorname{bOB}\left(n, p_{k}\right)$ on uniform crossover, which is the OB-Scan performing best in our experiments. The table exhibits that GAs using $\operatorname{bOB}\left(n, p_{k}\right)$ gain superiority in mean best fitness over GAs using uniform crossover by $62-89 \%$ on the F2e, $16-38 \%$ on the RAS, and $4-28 \%$ on the GRI functions. Moreover, we perform a one-tailed $t$-test to check the statistical significance. With confidence level $\alpha=0.05$, the $p$-values in Table III validate that $\operatorname{bOB}\left(n, p_{k}\right)$ using 2 to 10 parents is capable of significant improvement on uniform crossover in mean best fitness, except $\operatorname{bOB}\left(2, p_{k}\right)$ on the GRI function. The results also reveal that using more than two parents in $\operatorname{bOB}\left(n, p_{k}\right)$ achieves more significant improvement on uniform crossover. The significance, nevertheless, does not get greater absolutely with the increase of parents: In all the three test functions, even though the $p$-values decreases as the number of parents increases from 2 to 6 , the tendency of $p$-values becomes inconclusive for $\operatorname{bOB}\left(n, p_{k}\right)$ using more than 6 parents.

\section{B. Convergence Speed}

In terms of convergence speed, we first look into the influence of the number of parents on the convergence of GAs using $\mathrm{OB}-\mathrm{Scan}, \operatorname{bOB}\left(n, p_{k}\right)$, and $\operatorname{bOB}\left(n, 1-p_{k}\right)$, respectively. Due to the similarity of results on different problems, we present here the results on the F2e function only. Figure 3 demonstrates that the increase of parents has different effects on the convergence speed of GAs using these crossovers. In OB-Scan, increasing parents gains faster convergence in the very beginning, retards the convergence soon, and leads to a worse solution quality in the end. Such 
TABLE III

COMPARISON OF THE SOLUTION QUALITY BETWEEN UNIFORM CROSSOVER AND bOB $\left(n, p_{k}\right)$. THE $p$-VALUES REPRESENT THE $t$-TEST RESULTS

(SIGNIFICANT IMPROVEMENT OF $\mathrm{bOB}\left(n, p_{k}\right)$ ON UNIFORM CROSSOVER IS MARKED WITH BOLDFACE)

\begin{tabular}{|c|c|c|c|c|c|c|c|c|c|c|}
\hline \multirow[b]{2}{*}{ Crossover } & \multirow[b]{2}{*}{$n$} & \multicolumn{3}{|c|}{ F2e (opt: 0 ) } & \multicolumn{3}{|c|}{ RAS (opt: 0) } & \multicolumn{3}{|c|}{ GRI (opt: 0) } \\
\hline & & Avg. & Std. & $P$-value & Avg. & Std. & $P$-value & Avg. & Std. & $P$-value \\
\hline Uniform & 2 & 20.89 & 16.70 & & 4.30 & 2.81 & & 0.2386 & 0.0962 & \\
\hline $\mathrm{bOB}\left(n, p_{k}\right)$ & 2 & 8.01 & 8.26 & $2.34 \mathrm{E}-10$ & 3.61 & 2.12 & $2.55 \mathrm{E}-02$ & 0.2286 & 0.0822 & $2.15 \mathrm{E}-01$ \\
\hline & 3 & 4.75 & 4.96 & 2.18E-15 & 2.91 & 1.69 & 2.52E-05 & 0.2105 & 0.0746 & 1.14E-02 \\
\hline & 4 & 4.66 & 6.85 & 8.70E-15 & 3.23 & 1.82 & $9.21 \mathrm{E}-04$ & 0.1839 & 0.0659 & $4.36 \mathrm{E}-06$ \\
\hline & 5 & 3.69 & 5.00 & 1.07E-16 & 2.78 & 1.58 & 3.80E-06 & 0.1804 & 0.0629 & 9.48E-07 \\
\hline & 6 & 2.76 & 3.28 & $2.06 \mathrm{E}-18$ & 2.66 & 1.63 & $9.56 \mathrm{E}-07$ & 0.1776 & 0.0623 & 3.14E-07 \\
\hline & 7 & 3.46 & 5.56 & 8.86E-17 & 3.03 & 1.74 & 1.04E-04 & 0.1811 & 0.0745 & 3.73E-06 \\
\hline & 8 & 2.89 & 3.78 & 4.16E-18 & 2.94 & 1.81 & 4.56E-05 & 0.1717 & 0.0742 & 1.45E-07 \\
\hline & 9 & 2.33 & 3.28 & 5.78E-19 & 3.17 & 1.88 & 5.69E-04 & 0.1836 & 0.0731 & 7.58E-06 \\
\hline & 10 & 2.73 & 3.48 & 2.14E-18 & 2.78 & 1.73 & 6.11E-06 & 0.2095 & 0.1043 & 2.13E-02 \\
\hline
\end{tabular}

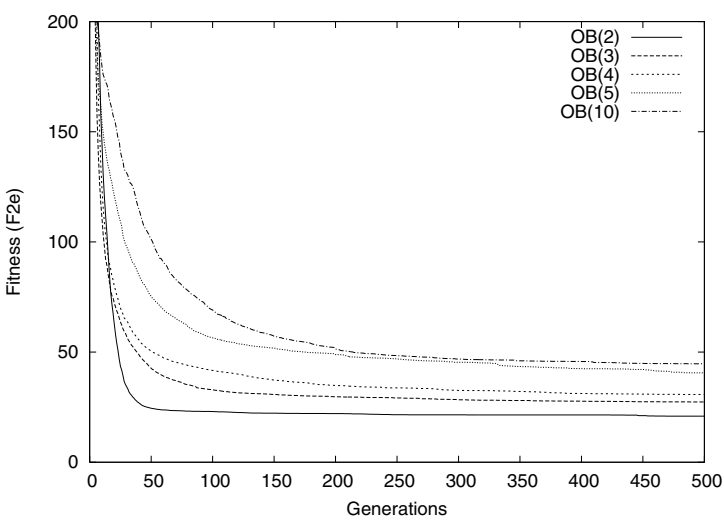

(a) $\mathrm{OB}-\mathrm{S} \operatorname{can}(n)$

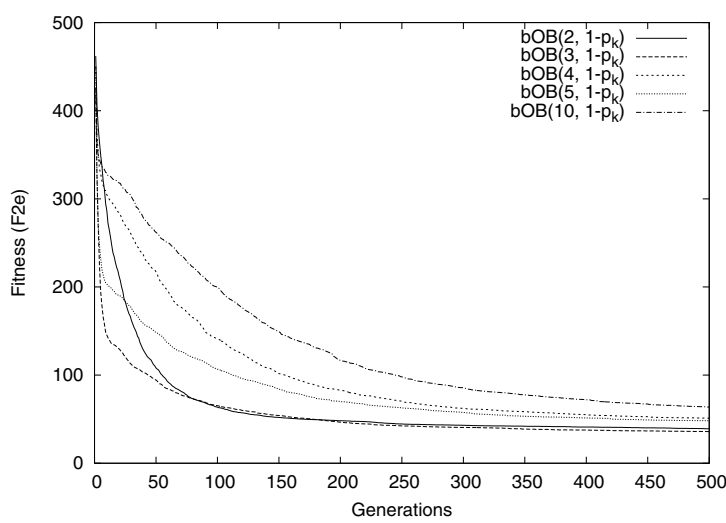

(b) $\operatorname{bOB}\left(n, 1-p_{k}\right)$

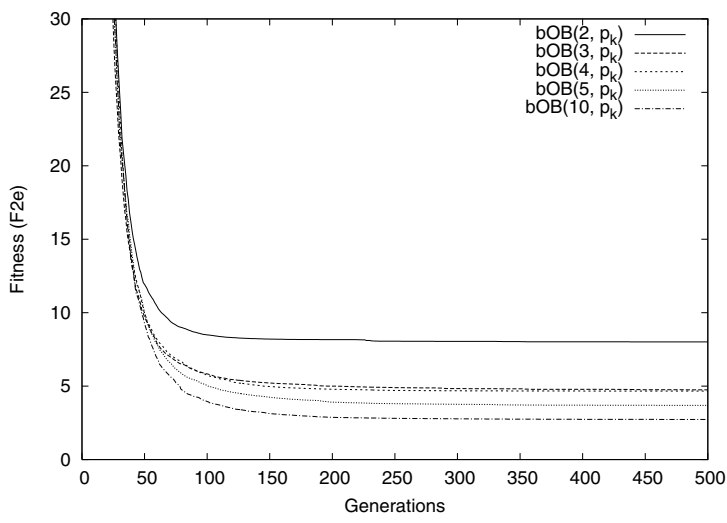

(c) $\operatorname{bOB}\left(n, p_{k}\right)$

Fig. 3. Convergence of GAs using (a) OB-Scan, (b) bOB $\left(n, 1-p_{k}\right)$, and (c) bOB $\left(n, p_{k}\right.$ ) with different number of parents on the F2e function (Note the different $y$-scales)

situation is a common symptom of premature convergence, caused by over-intensified exploitation. The $\operatorname{bOB}(n, 1-$ $p_{k}$ ) further aggravates this problem and consequently has even worse solution quality, compared to OB-Scan and $\operatorname{bOB}\left(n, p_{k}\right)$. However, $\operatorname{bOB}\left(n, p_{k}\right)$ can benefit from the increase of parents: Figure 3(c) shows that both convergence speed and solution quality improve with the number of parents, ranging from 2 to 10 . The preferable results confirm the merit of using more parents in the proposed $\operatorname{bOB}\left(n, p_{k}\right)$.
Next, we examine the impacts of crossover on the convergence speed of GAs. Figure 4 compares the convergence of GAs using $\mathrm{OB}-\mathrm{Scan}, \operatorname{bOB}\left(n, p_{k}\right)$, and $\operatorname{bOB}\left(n, 1-p_{k}\right)$ on the three test functions. Here the adopted number of parents for each crossover is the number that leads to the best solution quality respectively. The results in Fig. 4 show that $\operatorname{bOB}\left(n, p_{k}\right)$ outperforms both OB-Scan and $\operatorname{bOB}\left(n, p_{k}\right)$ in convergence speed as well as solution quality. Nonetheless, $\mathrm{GA}$ using $\operatorname{bOB}\left(n, 1-p_{k}\right)$ is inferior to that $\operatorname{using} \operatorname{bOB}\left(n, p_{k}\right)$ 


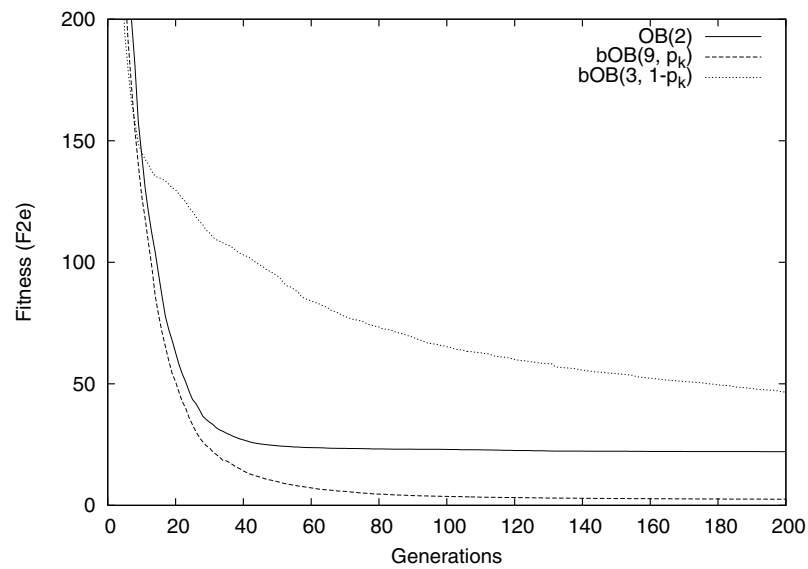

(a) $\mathrm{F} 2 \mathrm{e}$

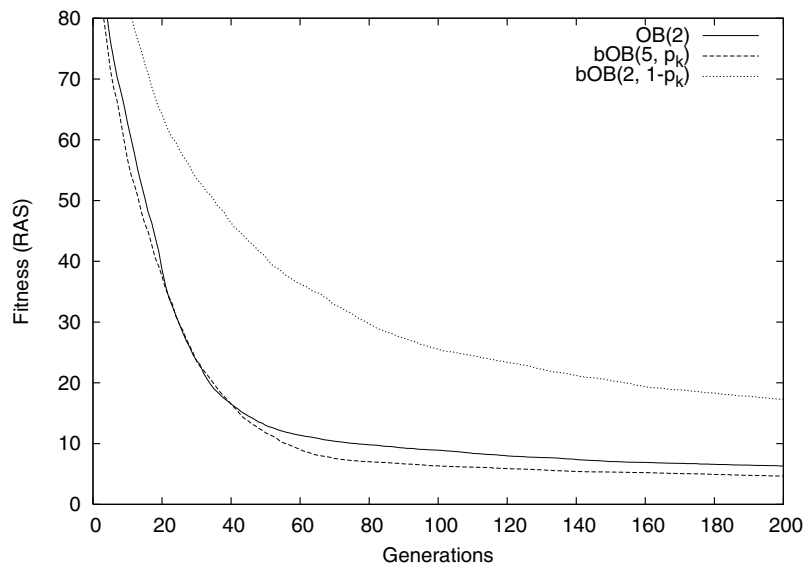

(b) RAS

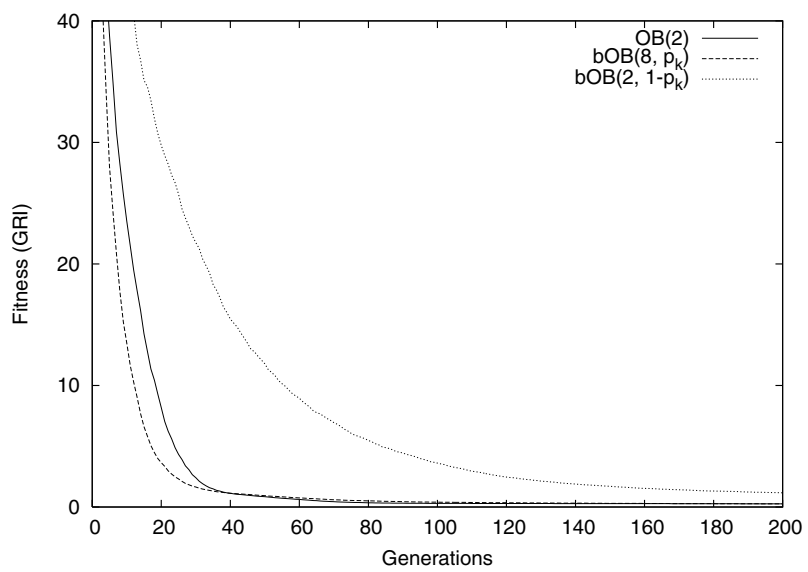

(c) GRI

Fig. 4. Comparison of convergence for GAs using OB-Scan, $\operatorname{bOB}\left(n, p_{k}\right)$, and $\mathrm{bOB}\left(n, 1-p_{k}\right)$ on (a) the F2e, (b) the RAS, and (c) the GRI functions

or OB-Scan. These outcomes reconfirm the effectiveness of supermajority strategy in control of exploitation and exploration in the multi-parent crossover: $\mathrm{bOB}\left(n, p_{k}\right)$ is highly capable of balancing the two forces but $\operatorname{bOB}\left(n, 1-p_{k}\right)$ exacerbates the problems of OB-Scan in lopsided intensification on exploitation.

\section{CONCLUSION}

The paper proposes the use of supermajority to deal with the issue of exploitation and exploration in multi-parent crossover. The proposed approach, called biased occurrencebased scanning crossover (bOB), determines offspring genes using the $\theta$-majority in place of the simple majority in OBScan. Through control over the threshold $\theta$, we are able to regulate the level of preference for major alleles in the crossover. Two variants of bOB crossover, $\operatorname{viz} \operatorname{bOB}\left(n, p_{k}\right)$ and $\operatorname{bOB}\left(n, 1-p_{k}\right)$, are developed on the basis of allele frequency $p_{k}$. These two crossovers determine offspring genes according to the adaptive majority thresholds $p_{k}$ and $1-p_{k}$, instead of fixed $\frac{1}{2}$ in the OB-Scan. Hence, $\operatorname{bOB}\left(n, p_{k}\right)$ makes major alleles more difficult while $\operatorname{bOB}\left(n, 1-p_{k}\right)$ makes them easier to be chosen as offspring genes.
Experimental results demonstrate the effects of the proposed crossovers on the performance of GAs. The 2-parent $\operatorname{bOB}\left(n, p_{k}\right)$ achieves significant improvement on $n$-parent OB-Scan with $n=2, \ldots, 10$ in terms of solution quality and convergence speed. Increasing the number of parents in $\operatorname{bOB}\left(n, p_{k}\right)$ further enhances the performance. In terms of mean best fitness, GAs using bOB $\left(n, p_{k}\right)$ outperform GAs using uniform crossover, i.e. the OB-Scan performing best in out experiments, by $62-89 \%$ on the F2e, $16-38 \%$ on the RAS, and $4-28 \%$ on the GRI functions. These preferable results of $\operatorname{bOB}\left(n, p_{k}\right)$ confirm the merits of supermajority in overcoming the issue of over-intensified exploitation in OB-Scan. The results also validate that the intensification of exploitation and exploration by the increase of parents in crossover can be advantageous - if they can be well controlled, as $\operatorname{bOB}\left(n, p_{k}\right)$ does. On the other hand, the experimental results exhibit that $\mathrm{bOB}\left(n, 1-p_{k}\right)$ suffers from premature convergence seriously, which reveals its excessive intensification on exploitation over OB-Scan.

Several directions remain for future work. First, the present work evaluates the performance of bOB crossover with merely three test functions. To validate the generality of 
bOB's advantages, experiments on more test functions should be carried out systematically. Second, bOB provides an effective way by adjusting the threshold $\theta$ to control exploitation and exploration in crossover. Therefore, it establishes a promising direction for future study on design of (multiparent) crossover for balance of the two driving forces and for enhancement of the performance of GAs. Work toward self-adaptive strategies and analysis on the influences of $\theta$ is underway.

\section{ACKNOWLEDGMENT}

This work was partially supported by the National Science Council of Taiwan.

\section{REFERENCES}

[1] T. Bäck and H.-P. Schwefel. An overview of evolutionary algorithms for parameter optimization. Evolutionary Computation, 1(1):1-23, 1993.

[2] A.E. Eiben. Multiparent recombination. In Evolutionary Computation 1: Basic Algorithms and Operators, pages 289-307. Institute of Physics Publishing, 2000.

[3] A.E. Eiben. Multiparent recombination in evolutionary computing. In Advances in Evolutionary Computing, pages 175-192. Springer, 2002.

[4] A.E. Eiben, P-E. Raué, and Zs. Ruttkay. Genetic algorithms with multi-parent recombination. In Parallel Problem Solving from Nature - PPSN III, volume 866 of LNCS, pages 78-87. Springer, 1994.

[5] A.E. Eiben and C.H.M. van Kemenade. Diagonal crossover in genetic algorithms for numerical optimization. Journal of Control and Cybernetics, 26(3):447-465, 1997.

[6] A.E. Eiben, C.H.M. van Kemenade, and J.N. Kok. Orgy in the computer: Multi-parent reproduction in genetic algorithms. In Proceedings of the Third European Conference on Artificial Life, volume 929, pages 934-945, 1995.

[7] J. Holland. Adaptation in Natural and Artificial Systems. University of Michigan Press, 1975.

[8] H. Kita, I. Ono, and S. Kobayashi. Multi-parental extension of the unimodal normal distribution crossover for real-coded genetic algorithms. In Proceedings of the Congress on Evolutionary Computation, volume 2, pages 1581-1588. IEEE Press, 1999.

[9] H. Mühlenbein, M. Schomisch, and J. Born. The parallel genetic algorithm as function optimizer. In Proceedings of the 4th International Conference on Genetic Algorithms, pages 271-278. Morgan Kaufmann, 1991.

[10] H.-P. Schwefel. Evolution and Optimum Seeking. John Wiley \& Sons, Inc., 1995.

[11] G. Syswerda. Uniform crossover in genetic algorithms. In Proceedings of the 3rd International Conference on Genetic Algorithms, pages 2-9. Morgan Kaufmann, 1989.

[12] C.K. Ting. On the convergence of multi-parent genetic algorithms. In Proceedings of the 2005 Congress on Evolutionary Computation, volume 1, pages 396-403. IEEE Press, 2005.

[13] C.K. Ting. On the mean convergence time of multi-parent genetic algorithms without selection. In Proceedings of the Eighth European Conference on Artificial Life, volume 3630 of LNAI, pages 403-412. Springer-Verlag, 2005.

[14] S. Tsutsui. Multi-parent recombination in genetic algorithms with search space boundary extension by mirroring. In Parallel Problem Solving from Nature - PPSN V, volume 1498 of Lecture Notes in Computer Science, pages 428-437. Springer, 1998.

[15] S. Tsutsui and A. Ghosh. A study on the effect of multi-parent recombination in real coded genetic algorithms. In Proceedings of International Conference on Evolutionary Computation, pages 828833, 1998.

[16] S. Tsutsui and L.C. Jain. On the effect on multi-parent recombination in real coded genetic algorithms. In Proceedings of the 2nd International Conference on Knowledge-based Intelligent electronic systems, pages 155-160, 1998.

[17] S. Tsutsui, M. Yamamura, and T. Higuchi. Multi-parent recombination with simplex crossover in real coded genetic algorithms. In Proceedings of the Genetic and Evolutionary Computation Conference, volume 1, pages 657-664. Morgan Kaufmann, 1999.
[18] H.-M. Voigt and H. Mühlenbein. Gene pool recombination and the utilization of covariances for the breeder genetic algorithm. In Proceedings of the Second IEEE International Conference on Evolutionary Computation, pages 172-177. IEEE Press, 1995. 